\title{
Modeling the solar cycles 12-20 with a Babcock-Leighton flux transport dynamo
}

\author{
Jie Jiang \\ Key Laboratory of Solar Activity, National Astronomical Observatories, \\ Chinese Academy of Sciences, Beijing 100012, China \\ email: jiejiang@nao.cas.cn
}

\begin{abstract}
We use a Babcock-Leighton-type of dynamo with the poloidal source based as closely as possible on the observations to model the solar cycle irregularities during the cycles 12-20. The nonlinearities in the poloidal field generation, i.e., the cycle dependence of the sunspot group tilt angles and the latitudes are included. The convective pumping dominates the transport of the surface poloidal field to the tachocline. Our results show that the modeled polar fields have a good correlation with the observed next cycle strength and the build-up of the toroidal flux at the tachocline is strongly correlated with the observed cycle strength as well. Both correlation coefficients are above 0.8 . The success of the model indicates that they are the nonlinearities in the poloidal field generation which cause the solar cycle irregularities.
\end{abstract}

Keywords. Sun: magnetic fields, Sun: activity, Sun: interior

\section{Introduction}

Recent analyses of the long-term sunspot observations suggest that the dynamo is of the Babcock-Leighton (BL) type (Dasi-Espuig et al. 2010, Cameron 2011 and Kitchatinov \& Olemskoy 2011), in which the poloidal field generation is due to the decay of the tilt sunspot groups near the solar surface. We have the detailed sunspot record including longitudes, latitudes, areas and tilt angles since 1874 onwards. In this work, we use a BL-type dynamo model with the poloidal source based as closely as possible on the observations to simulate the evolution of the subsurface magnetic field over solar cycles 12-20 and further to understand the reason to cause the solar cycle irregularities.

We study the time period during solar cycles 12-20 that Royal Greenwich Observatory recorded the sunspot groups with the consistent sunspot area definition. Mount Wilson and Kadaikanal observatories have the consistent sunspot group tilt angle records during 1906 and 1986. For the beginning 3 cycles, we use the anti-correlation between the cycle strength and sunspot group tilt angles (Dasi-Espuig et al. 2010) to derive the individual sunspot group tilt. Moreover, latitude distributions of sunspot groups present the anticorrelation with the cycle strength (Jiang et al. 2010). The cycle dependence of tilt angles and latitude distribution of the sunspot groups are two nonlinear mechanisms to modulate the poloidal field generation at the solar surface. In the dynamo model, the inward pumping is included which dominates the transport of the poloidal field source from the surface to the tachocline.

\section{Flux transport dynamo model}

The dynamo model is based on the induction equation for an azimuthally symmetric field. We use the same profiles of the differential rotation, meridional flow and diffusivity as were used in Cameron et al. (2012). The magnetic pumping extends to the base of the convection zone, albeit at a weaker level than in the near-surface layer. 


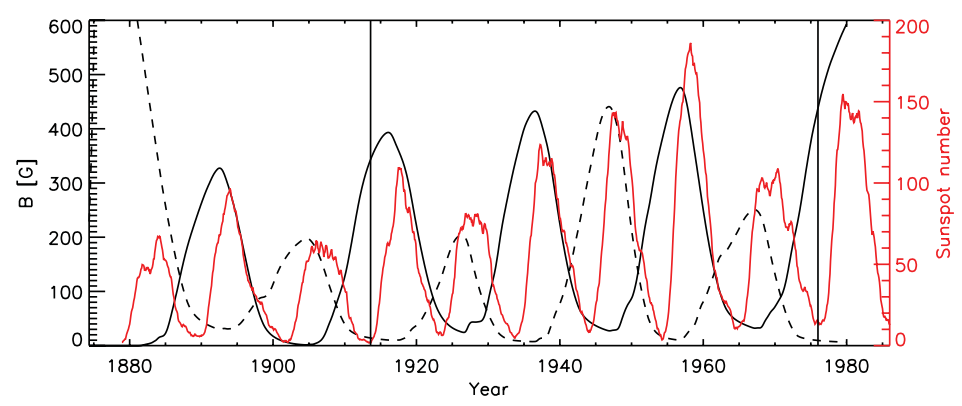

Figure 1. Time evolution of the modeled toroidal field at the base of the convection zone (black curves, solid and dashed curves corresponding to the alternating cycles) and the observed level of activity (red curve). The two vertical lines indicate the period for which cycle-averaged tilt angle observations are available. The toroidal flux associated with the first cycle is affected by the initial condition. The maxima of the modeled activity levels are clearly related to the amplitude of the observed cycle, the correlation coefficient being over 0.8 .

\section{Results}

The results indicate that both poloidal and toroidal fluxes have a simple form in that there are no remnants from previous cycles which is consistent with Jiang et al. (2007) and Karak \& Nandy (2012). The transport of poloidal flux from the surface to the tachocline occurs over the same time span as the flux is transported to the poles. The transport by downward pumping near the equator is opposed by the upward radial flow of the meridional circulation leading to a slower downwards transport near the equator, and a delayed reversal of the toroidal flux near the equator with respect to mid-latitudes. The surface evolution of the field is similar to that given by Cameron et al. (2010) who used the surface flux transport model. The correlation between the polar fields and the strength of the next cycle is above 0.8 . The equatorward propagating toroidal flux at low latitudes is derived. The evolution of the total toroidal flux at the base of the convection zone, between $r=0.65 R_{\odot}$ and $r=0.75 R_{\odot}$ and over latitudes $-45^{\circ}<\lambda<45^{\circ}$ is shown in Figure 1. The maxima of the modeled activity levels (black curves) are clearly related to the amplitude of the observed cycle (red curve), correlation coefficient being over 0.8.

We conclude that they are the nonlinearities of the poloidal field generation and hence the nonlinearities of the BL-type flux transport dynamo, i.e. the cycle-dependent tilt angles and latitudes of sunspot emergence which are responsible for the correlation between the modeled toroidal flux at the tachocline and the observed cycle strength. They are also the nonlinearities which cause the solar cycle irregularities.

The work is supported by the National Natural Science Foundations of China (11173033, 11178005, 11125314) and the Knowledge Innovation Program of CAS (KJCX2-EW-T07).

\section{References}

Cameron, R. H. 2011, in: A.R. Choudhuri \& D. Banerjee (eds.), First Asia-Pacific Solar Physics Meeting (ASI Conference Series), p. 143

Cameron, R. H., Jiang, J., Schmitt, D., \& Schüssler, M. 2010, ApJ, 719, 264

Cameron, R. H., Schmitt, D., Jiang, J., \& Işık, E. 2012, A\&A, 542, A127

Dasi-Espuig, M., Solanki, S. K., Krivova, N. A., Cameron, R. H., \& Peñuela, T. 2010, A\&A, 518, 7

Jiang, J., Cameron, R., Schmitt, D., \& Schüssler, M. 2010, A\&A, 528, 82

Jiang, J., Chatterjee, P., \& Choudhuri, A. R. 2007, MNRAS, 381, 1527

Karak, B. B. \& Nandy, D. 2012, ArXiv e-prints, 1206.2106

Kitchatinov, L. L. \& Olemskoy, S. V. 2011, Ap. Lett., 37, 656 